Radial and Nonradial Pulsations as Probes of Stellar Physics

ASP Conference Series, Vol. 259, 2002

C. Aerts, T.R. Bedding, \& J. Christensen-Dalsgaard, eds.

\title{
A Proposed Pulsation Driving Mechanism for $\gamma$ Doradus Variable Stars
}

\author{
J. A. Guzik ${ }^{1}$, A. B. Kaye, P. A. Bradley, A. N. Cox, \\ C. Neuforge-Verheecke
}

Los Alamos National Laboratory, Los Alamos, NM 87545-2345, USA

\section{P. B. Warner}

Dept. of Physics and Astronomy, Brigham Young U., Provo, UT 84602

\begin{abstract}
We propose a pulsation driving mechanism for $\gamma$ Doradus variable stars. This mechanism requires that the local convective timescale at the base of the envelope convection zone be comparable to or longer than the pulsation period. This mechanism explains both the red and blue edges of the $\gamma$ Dor instability strip. We predict that some candidate $\gamma$ Dor stars summarized by Handler (1999) may actually be too hot or too cool to be $\gamma$ Dor variables.
\end{abstract}

\section{Models and Driving Mechanism}

The $\gamma$ Doradus stars are a recently discovered class of main sequence pulsating variables lying near the red edge of the $\delta$ Scuti instability strip (Kaye et al., 1999; Zerbi \& Kaye, these proceedings). These stars pulsate in one to several modes with periods of 0.4 to $\sim 3$ days, believed to be gravity modes.

We tested the pulsation stability of $Z=0.02$ and $Z=0.03$ evolutionary models lying near the $\gamma$ Dor instability region using the nonradial code of Pesnell (1990). The results are shown in Fig. 1. The crosses in Fig. 1 mark the location of three $1.62 \mathrm{M}_{\odot} Z=0.03$ models, and triangles mark $1.45 \mathrm{M}_{\odot} Z=0.02$ models discussed by Guzik et al. (2000). The reddest $1.62 \mathrm{M}_{\odot}$ model has only one mode with growth rate negligibly larger than zero, so it is probably stable. The models were translated to the color-magnitude diagram using the calibration of Smalley \& Kupka (1997). We find excellent agreement between model predictions and observations for both the localized region of pulsational instability in the HR diagram, and the period range of unstable modes. Models with $T_{\text {eff }} \sim 6800-7200 \mathrm{~K}$ are predicted to be unstable to high-order $\ell=1$ and $2 g$-modes with periods of 0.5 to 2.6 days, with maximum linear growth rates $\sim 10^{-4}$ per period. Gravity modes with periods shorter than about 0.5 day are predicted to be pulsationally stable. The results are described in detail by Guzik et al. (2000).

The key to the pulsation driving is the presence of a relatively deep envelope convection zone, with temperatures at the base of 170,000 to $\sim 450,000 \mathrm{~K}$.

\footnotetext{
ie-mail: joy@lanl.gov
} 


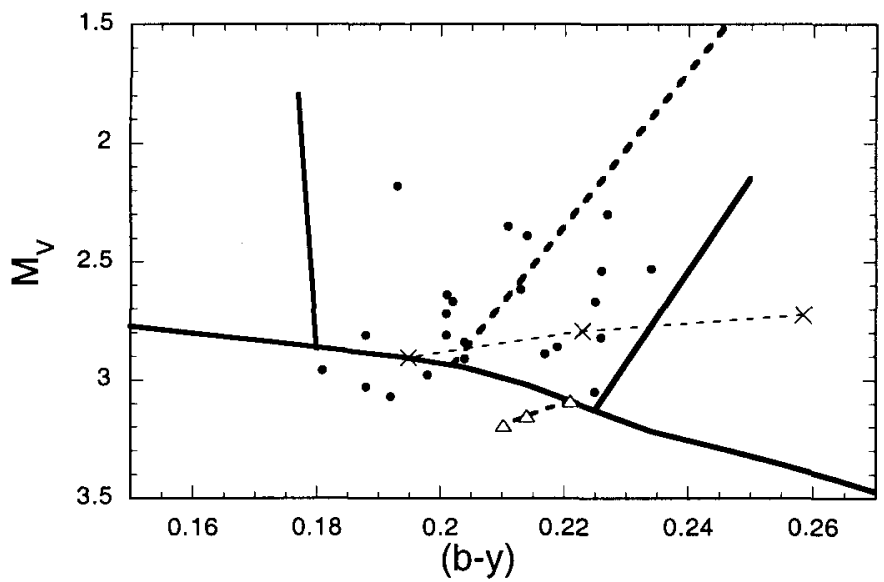

Figure 1. Filled circles mark bona fide $\gamma$ Dor stars, and solid lines mark revised proposed edges of the $\gamma$ Dor instability strip (Handler \& Shobbrook, 2002). The ZAMS location is from Crawford $(1975,1979)$. The dashed line is the red edge of the $\delta$ Sct instability strip (Rodriguez $\&$ Breger, 2001). For an explanation of the symbols $\mathrm{X}$ and $\triangle$ : see text.

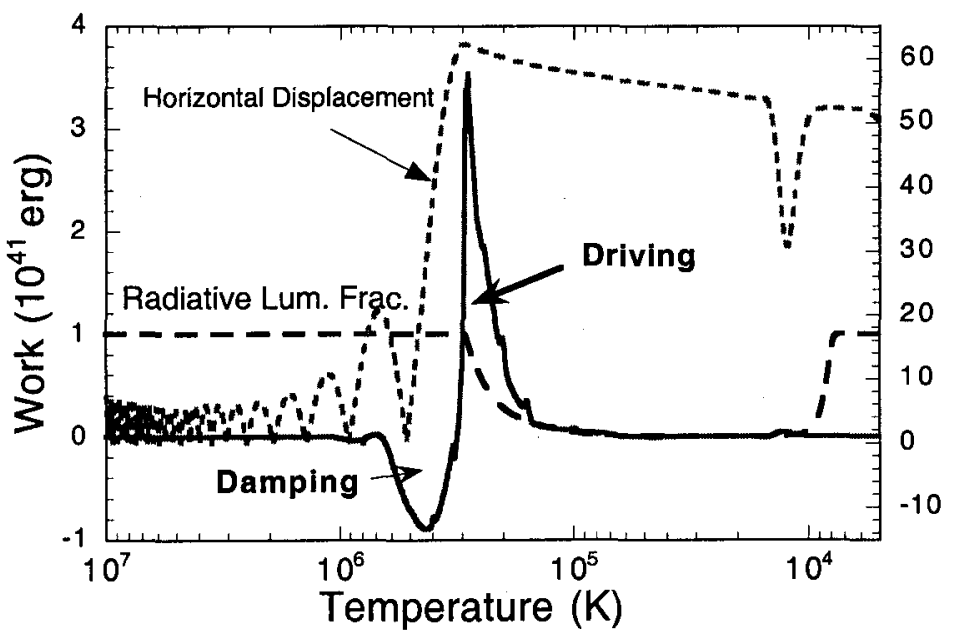

Figure 2. Work (solid line), luminosity fraction transported by radiation, and horizontal displacement modulus vs temperature for a $1.5 \mathrm{~d}$ mode with growth rate $2.3 \times 10^{-5}$ per period of the $1.62 \mathrm{M}_{\odot}$ model. See Guzik et al. (2000) for additional discussion. 


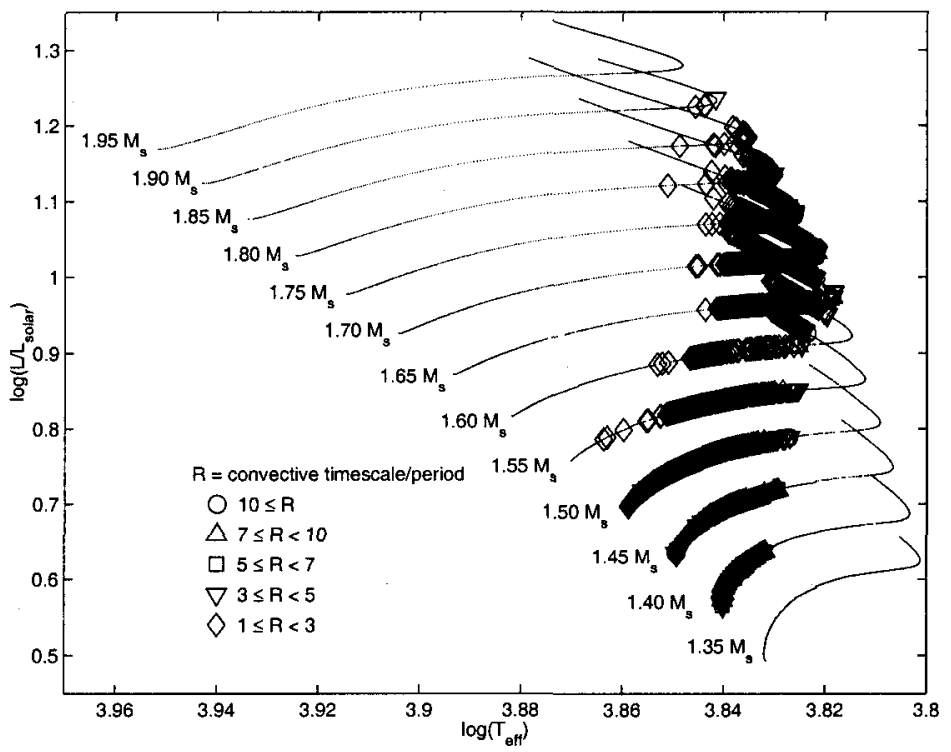

Figure 3. $Z=0.02$ models with unstable $\ell=1$ modes, growth rates $>10^{-7}$ per period, and a ratio of convective timescale at the $\mathrm{CZ}$ base to $g$-mode period greater than one.

At these temperatures, the local convective timescale (mixing length/local convective velocity) is comparable to or longer than the pulsation period, so the Pesnell code assumption of "frozen-in" convection, or zero fluctuations in convective luminosity during the pulsation cycle, is a valid approximation. When the convective timescale is long compared to the pulsation period, convection cannot adapt quickly enough during the pulsation cycle to transport the radiative flux emerging from the stellar center, and the flux is periodically blocked, resulting in driving (Fig. 2).

This mechanism predicts both edges of the instability strip: If the convection zone is too shallow, the convective timescale at the convection zone base becomes shorter than the pulsation period, so that convection can adapt to carry the flux. If, on the other hand, the convection zone becomes too deep, the fluctuations in radiative luminosity are smaller in these deeper, more nearly adiabatic layers, and so a smaller radiative fluctuation is being blocked, resulting in less driving, which can be overwhelmed by damping.

We are proceeding to map out the predicted $\gamma$ Dor instability strip. Figure 3 shows preliminary results by $\mathrm{P}$. Warner for the location of $Z=0.02$ models that have unstable $\ell=1$ modes with growth rates $>10^{-7}$ per period, and convective timescales at the $\mathrm{CZ}$ base longer than the pulsation period. These choices of growth rate and convective timescale cutoffs are somewhat arbitrary, pending model refinements to include the time dependence of convection and viscous 
damping, but serve to qualitatively place the red and blue edges of the instability region. The shape and size of this island of instability also depend somewhat on $\ell$; the instability region is slightly wider near the main sequence for $\ell=2$.

Modeling improvements are necessary to account for modulation of the convective flux, to investigate the effects of diffusive settling and radiative levitation, and to include possible additional damping due to shear viscosity. If our proposed pulsation mechanism is correct, then some candidate $\gamma$ Dor variables listed by Handler (1999) that are well outside the new Handler \& Shobbrook instability strip may actually be too hot or too cool to be $\gamma$ Dor stars.

Acknowledgments. We are especially grateful to G. Handler for providing updated data on confirmed $\gamma$ Dor stars prior to publication. We also acknowledge Y. Wu and H. Saio for useful discussions.

\section{References}

Crawford, D. 1975, AJ, 80, 955

Crawford, D. 1979, AJ, 84, 1858

Guzik, J.A., Kaye, A.B., Bradley, P.A., Cox, A.N., \& Neuforge, C. 2000, ApJ, $542, \mathrm{~L} 57$

Handler, G. 1999, MNRAS, 309, L19

Handler, G. \& Shobbrook, R.R. 2002, MNRAS, in preparation

Kaye, A.B., Handler, G., Krisciunas, K., Poretti, E., \& Zerbi, F. 1999, PASP, 111,840

Pesnell, W.D. 1990, ApJ, 363, 227

Rodriguez, E. \& Breger, M. 2001, A\&A, 366, 178

Smalley, B. \& Kupka, F. 1997, A\&A, 328, 349 